\title{
Polarization properties of second-harmonic generation in AIGaAs optical nanoantennas
}

\author{
L. Ghirardini, ${ }^{1}$ L. Carletti, ${ }^{2}$ V. Gill, ${ }^{3}$ G. Pellegrini, ${ }^{1}$ L. Duò, ${ }^{1}$ M. Finazzi, ${ }^{1}$ D. Rocco, ${ }^{2}$ A. Locatelli, ${ }^{2}$ \\ C. De Angelis, ${ }^{2}$ I. Favero, ${ }^{3}$ M. Ravaro, ${ }^{3}$ G. Leo,${ }^{3}$ A. Lemaître, ${ }^{4}$ and M. Celebrano ${ }^{1, *}$ \\ ${ }^{1}$ Department of Physics, Politecnico di Milano, Piazza Leonardo Da Vinci 32, 20133 Milano, Italy \\ ${ }^{2}$ Department of Information Engineering, University of Brescia, Via Branze 38, 25123 Brescia, Italy \\ ${ }^{3}$ Matériaux et Phénomènes Quantiques, Université Paris Diderot-Sorbonne Paris Cité, 10 rue A. Domon et L. Duquet, 75013 Paris, France \\ ${ }^{4}$ Centre de Nanosciences et de Nanotechnologies, CNRS-UMR9001, Route de Nozay, 91460 Marcoussis, France \\ ${ }^{*}$ Corresponding author: michele.celebrano@polimi.it
}

\begin{abstract}
Manipulating light at the nanoscale by means of dielectric nanoantennas recently received renewed attention thanks to the development of key enabling fabrication tools in semicon-ductor technology, combined with the extremely low losses exhibited by dielectrics in the optical regime. Nanostructures based on III-V type semiconductors, characterized by an intrinsic broken symmetry down to a single elementary cell, has already demonstrated remarkable nonlinear conversion efficiencies at scales well below the operating wavelength. In this Letter, we thoroughly investigate the emission proper-ties of second-harmonic generation (SHG) in AlGaAs mono-lithic nanoantennas. Our findings point toward the pivotal role of volume susceptibility in SHG, further unraveling the physics behind the nonlinear processes in these systems. The extremely high SHG efficiency attained, together with the control over the polarized emission in these nanoantennas, constitute key ingredients for the development of tunable nonlinear metasurfaces.
\end{abstract}

High-permittivity semiconductor nanoparticles are emerging as a promising platform for nanophotonics due to their potential to manipulate and route light at sub-wavelength scales [1-4]. The capability of concentrating the optical field in highly confined volumes with negligible absorption makes these objects particularly attractive for nonlinear nanophotonic applications. This prompted the investigation of third-harmonic generation from both $\mathrm{Si}$ [5-7] and Ge [8] nanostructures, with reported nonlinear signal enhancements ranging from two to five orders of magnitude compared to an unstructured film, respectively. Since these semiconductors are centrosymmetric, second-order nonlinear effects within the material volume are forbidden. Conversely, in zinc-blend nanostructures, these processes can be considerably enhanced. In this context, widespread III-Vlaser materials like GaAs and AlGaAs recently gained a great deal of interest due to their large $\chi^{(2)}$ value [9-13]. AlGaAs is particu-larly interesting for second-harmonic generation (SHG) in the telecom range. Since its energy bandgap increases with the $\mathrm{Al}$ content, the optical transparency window can be extended at both the pump (fundamental frequency, FF) and the second har-monic (SH) frequency, and two-photon absorption is suppressed at the pump wavelength. This is obviously beneficial in terms of higher SHG conversion efficiency and damage threshold.
SHG conversion efficiency in AlGaAs monolithic nanodisks suspended in air was numerically predicted to be as high as $10^{-3}$ for a pump excitation centered at $1.55 \mu \mathrm{m}$, with an intensity of about $1.6 \mathrm{GW} / \mathrm{cm}^{2}$ [9]. Recently, we experimentally demonstrated efficient SHG from the same nanoscale system laying on an aluminum-oxide (AlOx) substrate, detected in reflection geometry.

Using the same pump parameters as Ref. [9], we experimentally determined a nonlinear conversion efficiency exceeding 10 -5 [10], which is lower than the predicted one due to the presence of the substrate, which smears the quality factor of the nanocavity. Yet, this value is more than 3 orders of magni-tude higher than the values reported in the literature for SHG in plasmonic nanostructures working in the telecom range with comparable lateral fingerprints and investigated under the same experimental conditions [14]. Similar results in terms of efficiency have been reported concurrently from a GaAs-on-AlOx monolithic metasurface [11]. SHG has also been achieved in AlGaAs nanoantennas embedded in a completely transparent substrate (benzo-cyclobutane, $\mathrm{BCB}$ ), both in reflection and in transmission, showing nontrivial polarization features [12]. Because of the intriguing potential applications of these SHG nanostructures to, e.g., nonlinear holography [15] and background-free wave-shaping metasurfaces [16], the richness of their polarization behavior deserves further investigation.

In this Letter, we focus on the polarization properties of SHG in individual monolithic AlGaAs-on-AlOx nanoantennas. Our samples consist of isolated ( $3 \mu \mathrm{m}$ spacing) $\mathrm{Al}_{0.18} \mathrm{Ga}_{0.82} \mathrm{As}$ nanodisks, with height $h=400 \mathrm{~nm}$ and radii ranging from

This is the accepted version of Ghirardini L., Carletti L., Gili V., Pellegrini G., Duò L., Finazzi M., Rocco D., Locatelli A., De Angelis C., Favero I., Ravaro M., Leo G., Lemaître A., Celebrano M., "Polarization properties of second-harmonic generation in AlGaAs optical nanoantennas", Optics Letters, 2017, 42:3, 559-562.

The final publication is available at: https://doi.org/10.1364/OL.42.000559 


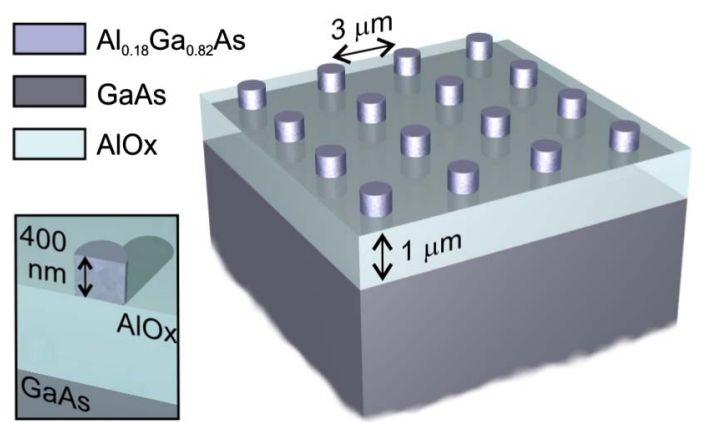

Fig. 1. Schematic view of the AlGaAs-on-AlOx nanodisk array [9].

175 to $225 \mathrm{~nm}$, on a $1 \mu \mathrm{m}$-thick AlOx substrate. Such a thick and relatively low refractive index substrate allows us to effectively decouple the nanocavity modes from the underlying GaAs (100) wafer. The design and fabrication are detailed in Ref. [10], while a sketch of the structure is presented in Fig. 1.

Here, we study the emission properties of the resonantly enhanced SHG from these AlGaAs nanoantennas, which are transparent at both the pump and $\mathrm{SH}$ wavelengths, by performing polarization-resolved measurements. To this end, we inserted a polarizer in the SHG collection path of the same experimental setup described in Ref. [10], which consists of a nonlinear microscope coupled with a linearly polarized (extinction ratio $>100: 1)$ ultrafast laser (150 fs pulse width) centered at $1.55 \mu \mathrm{m}$. To access the various resonances involved in the process and retrieve spectral information over the system using a fixed excitation wavelength, we analyzed nanoantennas with different radii spanning from 175 to $225 \mathrm{~nm}$. This approach is ideal to optimally access the nonlinear properties of these cylindrical nanocavities, since it ensures optical transparency at both the pump and emission wavelengths. Moreover, both the dispersion of the material refractive index and the chromatic aberration in the experimental setup can be neglected. Yet, our results can be still compared with the ones employing a tunable pump [11] since (i) all the relevant phenomena occur at energies well below the material resonances, [17] and (ii) other experimental realizations are conducted on relatively narrow spectral ranges, which implies a very limited refractive index dispersion [18]. Therefore, all the meaningful physics are expected to scale as the radius-to-wavelength ratio $r / \lambda$.

In our experiment, we focus a linearly polarized pump beam on the sample using a high-numerical-aperture $(\mathrm{NA}=0.85)$ air objective. Figure 2(a), showing the SHG conversion efficiency of our platforms plotted as a function of $r / \lambda$, reveals two resonant peaks around 0.123 and 0.140 . The absolute calibration of the efficiency displayed on the left vertical axis of the graph is experimentally derived from the total photon flux (on the right vertical axis) that is estimated by accounting for the losses in the optical collection path of the experimental setup (see Ref. [10]). The overall photon flux from the brightest nanodisk is up to about $3 \times 10^{10} \mathrm{~s}^{-1}$, which corresponds to a maximal conversion efficiency $\eta_{\mathrm{SHG}} \cong 10^{-5}$ and to a nonlinear coefficient $\beta_{\mathrm{SHG}}=\hat{P}_{\mathrm{SH}} / \hat{P}_{\mathrm{FF}}^{2} \cong 1.5 \times 10^{-7} \mathrm{~W}^{-1}$, with $\hat{P}_{\mathrm{SH}}$ and $\hat{P}_{\mathrm{FF}}$ as the peak powers estimated for the $\mathrm{SH}$ emission and the FF illumination, respectively. While the reported SHG conversion efficiency is comparable with the one recently reported from similar nanoscale systems [11], the nonlinear coefficient of our nanoscale systems is up to one order of magnitude higher. This is due to the
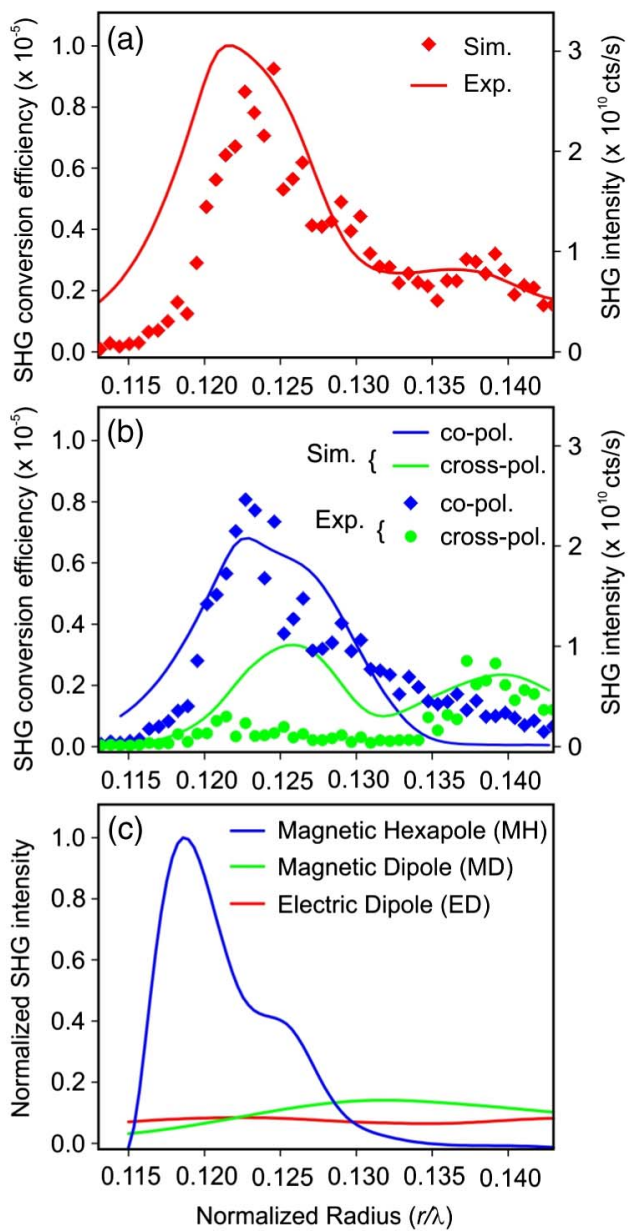

Fig. 2. (a) Simulated (line) and experimental (diamonds) unpolarized emission as a function of the normalized radius, $r / \lambda$. Results are plotted both as overall photon count and as equivalent conversion efficiency. (b) Calculated (lines) and experimental (dots) polarized emission as a function of $r / \lambda$. Co- and cross-polarized SHG are plotted in blue and green, respectively. (c) Multipole decomposition of the fields emitted at the $\mathrm{SH}$ wavelength as a function of $r / \lambda$.

lower pump flux applied, which is limited in our work by the saturation of the detector occurring with the higher SHG signal level. As already described in Ref. [10], efficient optical pumping of the structure is provided by the resonant excitation of an electric and a magnetic dipolar mode at the pump wavelength. However, as also reported in Ref. [12], the relatively narrow features observed in the experimentally recorded $\mathrm{SH}$ signal as a function of the nanodisk radius [see Fig. 2(a)] are more likely to be associated with multipolar electric and magnetic resonances characterizing the nanoantenna in the SH spectral range.

When analyzing the polarization of the emitted SHG in our nanostructured AlGaAs, a significant variation is observed in the polarization state of the $\mathrm{SH}$ light as a function of the nanodisk radius, as shown by the dotted plots in Fig. 2(b). The SH light emitted by nanoantennas with a smaller radius $(r / \lambda \approx 0.123)$ is mainly co-polarized with the pump beam, while the emission from nanoantennas featuring a larger radius $(r / \lambda \approx 0.140)$ is orthogonally polarized with respect to the pump. In optically isotropic AlGaAs, which belongs to the $\overline{4} 3 \mathrm{~m}$ symmetry group, the only nonzero elements of the bulk 
second-order nonlinear susceptibility tensor are of the type $\chi_{x y z}^{(2)}$, corresponding to the $d_{14}$ element in the Voigt contracted form. Therefore, an incident plane wave with its electric field that is linearly polarized along the bisector of two crystalline axes, say, [100] and [010], generates SH radiation that will be linearly polarized along the [001] direction in bulk AlGaAs. Given the rather complex composition of the illuminating beam that is required to access the individual nanodisks (i.e., a tightly focused Gaussian beam), this hand-waving mechanism cannot be easily compared with the experimental observations on the $\mathrm{SH}$ polarized emission represented by the dots in Fig. 2(b). Therefore, to gain insight into the mechanism underlying the polarized emission in these systems, we resort to fully vectorial finite-element numerical calculations in the nonlinear electromagnetic regime, using the same approach as in Ref. [9]. In our simulations, the nanodisks are placed on a substrate with a refractive index equal to 1.6, the linear optical dispersion of AlGaAs is modeled according to Ref. [18], and only the bulk $\chi^{(2)}$ is taken into account. In addition, to closely mimic the experimental conditions, the excitation is modeled as a focused Gaussian beam of about a $2 \mu \mathrm{m}$ waist, and the SHG signal is collected in reflection by integrating over a 0.85 NA. The simulated SHG conversion efficiency, shown in Fig. 2(a) as a red line superimposed onto the experimental data, shows excellent qualitative and quantitative agreement with the measured one.

By selecting field components that are co- and cross-polarized with respect to the pump field, we can model the polarization state of the $\mathrm{SH}$ light represented by the dots in Fig. 2(b). As can be noticed, the agreement between the simulations and our experimental data (see solid lines and dots in Fig. 2(b), respectively) is very good. All the relevant features in the two polarized emission plots are well matched, with only the peak in the crosspolarized emission at lower $r / \lambda$ values being stronger in the simulations than in the experiments. This deviation, as well as the signal oscillations in the experimental curves in both Figs. 2(a) and 2(b), is attributed to residual fabrication tolerances, which can lead to significant modification of the polarization state of the emitted $\mathrm{SH}$ at the individual nanoantenna level. In particular, electron diffraction images (not shown here) helped identify the presence of a random tilt in some nanodisks with respect to the surface normal, which can be up to $5^{\circ}$ and implies a local re-orientation of the susceptibility tensor at the individual nanoantenna level. This can be ascribed to an asymmetric
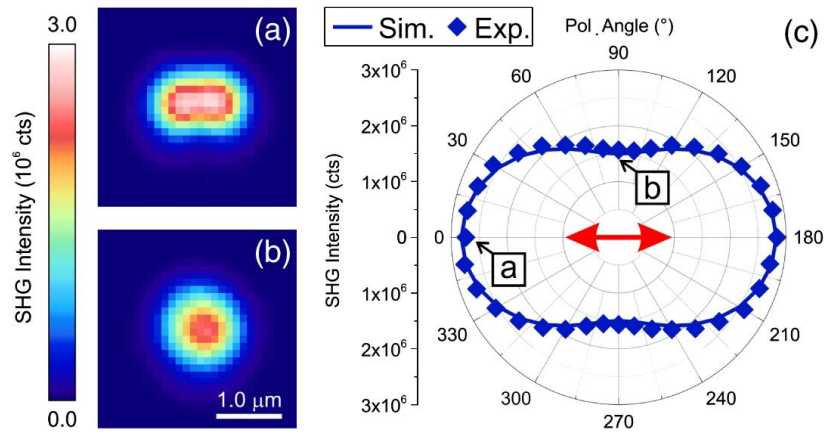

Fig. 3. Confocal SHG maps acquired on a $r / \lambda \approx 0.123$ nanodisk for emission polarization parallel [co-polarized, (a)] and perpendicular [cross-polarized, (b)] to the pump field. (c) Simulated (solid line) and experimentally acquired (diamonds) polar plots of the emitted $\mathrm{SH}$ from the nanostructure. Red arrow: pump polarization direction. Boxed letters: SHG values corresponding to the images on the left. sinking of the nano-object during the substrate oxidation. A detailed investigation of this phenomenon, however, goes beyond the scope of this Letter and will be addressed in a separate work.

To gain further insight into the modes involved in the emission processes, we investigated the different resonances that characterize the SHG emission peaks through a multipolar decomposition of the currents generated inside the resonator at the SH wavelength [19]. Figure 2(c) shows the main contributions to the $\mathrm{SH}$ signal as a function of $r / \lambda$. The emission enhancement occurring at lower $r / \lambda$ is dominated by a strong higher-order magnetic hexapolar resonance, while the secondary peak is mainly due to the overlap between the first-order electric and magnetic dipoles [9]. The magnetic quadrupole contribution here is negligible, due to the specific values of the disk sizes and the wavelength employed. The system, however, is very sensitive to these parameters, and in slightly different conditions, the quadrupolar contributions can become dominant and the hexapole contributions negligible. Since the computational model reported in Fig. 2(c) describes the fields emitted in the whole space, both the position and the spectral width of the emission peaks slightly deviate from the results reported in both Figs. 2(a) and 2(b), which are obtained by considering only the collection angle of the experimental setup.

Figure 3 shows the polarized $\mathrm{SH}$ emission from the smaller resonant nanodisks $(r / \lambda \approx 0.123)$. The experimental polar plot (blue dots) is extrapolated from a series of SHG confocal maps on individual nanodisks [see Figs. 3(a) and 3(b)], acquired while changing the angular position of the polarizer in the collection path. To account for possible anomalies induced by local imperfections in the structures, we averaged the overall $\mathrm{SH}$ emission collected from four different maps acquired on nominally identical individual nanoantennas. The shape of the SHG spot in Fig. 3(a), which is far from being Gaussian, indicates that optimal SHG with polarization parallel to the impinging one can only be achieved through side coupling with the structure. In contrast, SHG with polarization orthogonal to the pump beam is less efficient and is best excited when the pump beam is focused on the center of the nanodisks. Figure 3(c) shows the striking agreement between the measurements and simulations, both indicating that the radiated SHG is polarized mainly parallel to the pump polarization.

The polarized $\mathrm{SH}$ emission from the nanodisks with $r / \lambda=0.140$, analyzed using the same approach as in Fig. 3,
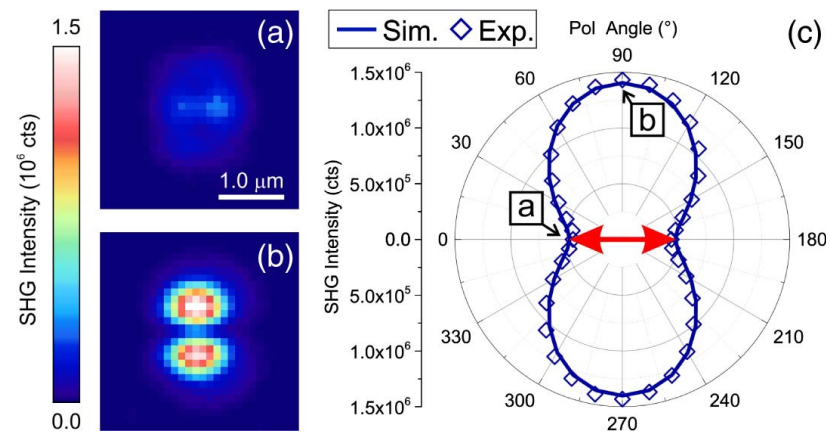

Fig. 4. Confocal SHG maps acquired on a $r / \lambda \approx 0.140$ nanodisk for emission polarization parallel [co-polarized, (a)] and perpendicular [cross-polarized, (b)] to the pump field. (c) Simulated (solid line) and experimentally acquired (diamonds) polar plots of the emitted $\mathrm{SH}$ from the nanostructure. Red arrow: pump polarization direction. Boxed letters: SHG values corresponding to the images on the left. 
is shown in Fig. 4. Even in this case, the agreement between the experimental data and simulation is excellent [see Fig. 4(c)], showing that the emitted $\mathrm{SH}$ light has a polarization state that is orthogonal to the pump. The remarkable matching between the experiments and calculations further supports our initial assumption about the cavity modes that dictates the polarization state of the emitted SHG by the nanoantennas. For crosspolarized emission, similar to the case of the smaller-radius nanodisks, optimized SHG is obtained when coupling to the side of the nanoantenna [see Fig. 4(b)]. It can be observed that in this last case, while the overall SH emission is lower, the degree of polarized emission is higher than for the nanoantennas displayed in Fig. 3. This fully agrees with the simulation plotted in Fig. 2(b), and it further confirms that the deviation between the measured and calculated polarized SHG reported in Fig. 2(b) can be attributed to fabrication tolerances in each individual nanodisk, which affect its $\mathrm{SH}$ emission polarization.

It is worth pointing out that our assumption about the bulk character of $\chi^{(2)}$ evidently suffices to explain the nonintuitively small dependence of the overall SHG collected power on the angle $\theta$ between the linearly polarized pump and a dielectric axis of AlGaAs. As a matter of fact, the excellent agreement between the measurements and the full-wave numerical simulations allows us to rule out the contribution of surface $\chi^{(2)}$ in this nanoscale system. This conclusion is in very good agreement with the experimental evidence reported in Ref. [12], as opposed to a recent claim concerning similar nanoscale platforms [11]. However, it is worth stressing that this behavior is strongly affected by the nature of the multipolar resonances in the SH spectral range, which depends on several parameters. A crucial issue, e.g., is the nanoantenna losses (at both FF and $\mathrm{SH})$ that are negligible in both the experiment reported here and in those of Refs. [12,13], as opposed to what happens in Ref. [11]. Moreover, the observed polarization is very sensitive to the illumination geometry; hence, the conversion efficiency is considerably reduced when illuminating all the nanodisks at once or arrays of nanodisks at normal incidence, e.g., by means of nondiffraction-limited pump beams. This behavior is far from being unexpected, since, although all experimental realizations to date refer to the case of normal incidence of the pump, a more complex phenomenology was predicted in the case of oblique incidence [20].

In conclusion, we investigated SHG in AlGaAs monolithic nanoantennas, analyzing its polarization properties in detail. This nanoscale dielectric nonlinear platforms provide a nonlinear coefficient, $\beta_{\mathrm{SHG}}$, that is one order of magnitude higher than those reported to date. The polarization-resolved measurements show a strong dependence between the $\mathrm{SH}$ emission polarization and the nanoantenna modes at the $\mathrm{SH}$ wavelength. Moreover, taking into account only the volume nonlinear susceptibility in the numerical simulations, we could accurately reproduce our experimental findings. This suggests a negligible contribution of surface nonlinearity to the SHG. Our results allow us to unravel the mechanisms underlying the polarized nonlinear emission in these highly nonlinear and transparent AlGaAs-on-insulator all-dielectric nanoantennas, showing the potential of these platforms in the manipulation of nonlinear light properties at the nanoscale.
Funding. European Cooperation in Science and Technology (COST) (NanoSpectroscopy COST Action MP1302); Fondazione Cariplo (Project 2013-0736); Education, Audiovisual and Culture Executive Agency (EACEA) (Erasmus Mundus NANOPHI (2013 5659/002-001)).

Acknowledgment. The authors warmly thank Prof. P. Biagioni, Prof. D. Neshev, and Prof. A. Zayats for the fruitful discussions and acknowledge the experimental support from Prof. C. Ricolleau and Dr. G. Wang.

\section{REFERENCES}

1. A. E. Krasnok, A. E. Miroshnichenko, P. A. Belov, and Y. S. Kivshar, Opt. Express 20, 20599 (2012).

2. M. Celebrano, M. Baselli, M. Bollani, J. Frigerio, A. Bahgat Shehata, A. Della Frera, A. Tosi, A. Farina, F. Pezzoli, J. Osmond, X. Wu, B. Hecht, R. Sordan, D. Chrastina, G. Isella, L. Duò, M. Finazzi, and P. Biagioni, ACS Photon. 2, 53 (2015).

3. S. Jahani and Z. Jacob, Nat. Nanotechnol. 11, 23 (2016).

4. M. Caldarola, P. Albella, E. Cortés, M. Rahmani, T. Roschuk, G. Grinblat, R. F. Oulton, A. V. Bragas, and S. A. Maier, Nat. Commun. 6, 7915 (2015).

5. M. R. Shcherbakov, D. N. Neshev, B. Hopkins, A. S. Shorokhov, I. Staude, E. V. Melik-Gaykazyan, M. Decker, A. A. Ezhov, A. E. Miroshnichenko, I. Brener, A. A. Fedyanin, and Y. S. Kivshar, Nano Lett. 14, 6488 (2014).

6. M. R. Shcherbakov, A. S. Shorokhov, D. N. Neshev, B. Hopkins, I. Staude, E. V. Melik-Gaykazyan, A. A. Ezhov, A. E. Miroshnichenko, I. Brener, A. A. Fedyanin, and Y. S. Kivshar, ACS Photon. 2, 578 (2015).

7. Y. Yang, W. Wang, A. Boulesbaa, I. I. Kravchenko, D. P. Briggs, A. Puretzky, D. Geohegan, and J. Valentine, Nano Lett. 15, 7388 (2015).

8. G. Grinblat, Y. Li, M. P. Nielsen, R. F. Oulton, and S. A. Maier, Nano Lett. 16, 4635 (2016).

9. L. Carletti, A. Locatelli, O. Stepanenko, G. Leo, and C. De Angelis, Opt. Express 23, 26544 (2015).

10. V. F. Gili, L. Carletti, A. Locatelli, D. Rocco, M. Finazzi, L. Ghirardini, I. Favero, C. Gomez, A. Lemaître, M. Celebrano, C. De Angelis, and G. Leo, Opt. Express 24, 15965 (2016).

11. S. Liu, S. Saravi, G. A. Keeler, M. B. Sinclair, Y. Yang, J. Reno, T. Pertsch, and I. Brener, Nano Lett. 16, 5426 (2016).

12. R. Camacho, M. Rahmani, S. Kruk, L. Wang, L. Xu, D. A. Smirnova, A. Solntsev, A. E. Miroshnichenko, H. H. Tan, F. Karouta, S. Naureen, K. Vora, L. Carletti, C. De Angelis, C. Jagadish, Y. S. Kivshar, and D. N. Neshev, Nano Lett. 16, 7191 (2016).

13. L. Carletti, D. Rocco, A. Locatelli, C. De Angelis, V. F. Gili, M. Ravaro, I. Favero, G. Leo, M. Finazzi, L. Ghirardini, M. Celebrano, G. Marino, and A. V. Zayats, "SHG at the nanoscale with monolithic AIGaAs-onAIOx antennas," Nanotechnology (to be published).

14. M. Celebrano, X. F. Wu, M. Baselli, S. Grossmann, P. Biagioni, A. Locatelli, C. De Angelis, G. Cerullo, R. Osellame, B. Hecht, L. Duò, F. Ciccacci, and M. Finazzi, Nat. Nanotechnol. 10, 412 (2015).

15. E. Almeida, O. Bitton, and Y. Prior, Nat. Commun. 7, 12533 (2016).

16. S. Keren-Zur, O. Avayu, L. Michaeli, and T. Ellenbogen, ACS Photon. 3, 117 (2016).

17. M. El Allali, C. B. Soerensen, E. Veje, and P. Tidemand-Petersson, Phys. Rev. B 48, 4398 (1993).

18. S. Gehrsitz, F. K. Reinhart, C. Gourgon, N. Herres, A. Vonlanthen, and H. Sigg, J. Appl. Phys. 87, 7825 (2000).

19. P. Grahn, A. Shevchenko, and M. Kaivola, New J. Phys. 14, 093033 (2012).

20. L. Carletti, A. Locatelli, D. N. Neshev, and C. De Angelis, ACS Photon. 3, 1500 (2016). 